\title{
ANÁLISE DA RESPOSTA DA FREQÜÊNCIA CARDÍACA DURANTE A REALIZAÇÃO DE EXERCÍCIO ISOCINÉTICO EXCÊNTRICO DE GRUPAMENTO EXTENSOR DE JOELHO
}

\author{
Malfatti CA ${ }^{1}$, Rodrigues SY ${ }^{1}$, Takahashi ACM ${ }^{1}$, Silva E ${ }^{2}$, Menegon FA ${ }^{1}$, Mattiello-Rosa SM ${ }^{3}$, \\ Catai AM ${ }^{1}$ \\ ${ }^{1}$ Núcleo de Pesquisa em Exercício Físico, Laboratório de Fisioterapia Cardiovascular, Universidade Federal de São \\ Carlos - UFSCar, São Carlos, SP \\ ${ }^{2}$ Faculdades de Ciências da Saúde, Curso de Fisioterapia, Universidade Metodista de Piracicaba, Piracicaba, SP \\ ${ }^{3}$ Laboratório de Patologia Experimental e Desempenho Humano, Departamento de Fisioterapia, UFSCar, São Carlos, SP \\ Correspondência para: Aparecida Maria Catai, Laboratório de Fisioterapia Cardiovascular - NUPEF, UFSCar, Rodovia \\ Washington Luís, km 235, CEP 13565-905, São Carlos, SP, e-mail: mcatai@power.ufscar.br
}

Recebido: 17/12/2004 - Aceito: 31/08/2005

\begin{abstract}
RESUMO
Objetivo: Investigar a magnitude da resposta da freqüência cardíaca durante o exercício isocinético excêntrico do grupamento extensor do joelho, em diferentes velocidades angulares. Materiais e Métodos: Dez voluntários jovens, sadios e ativos foram submetidos a contração excêntrica máxima do grupo extensor do joelho dominante. Foi utilizado um dinamômetro isocinético, nas velocidades de $30 \% \mathrm{~s}, 60 \% \mathrm{~s}$ e $120 \%$, ordenadas aleatoriamente. A FC foi obtida batimento a batimento, na derivação eletrocardiográfica MC5 modificada, durante 60s pré-exercício, durante o tempo de contração, sendo cinco repetições para cada velocidade, e por 120s após o esforço. Foram calculadas: FC média dos 60s pré-exercício, FC pico atingida ao final do esforço, e variação da FC $(\Delta \mathrm{FC})$, além dos valores do pico de torque. Também foram comparados valores da FC média dos 6 minutos préexercício com os 6 minutos de recuperação. Os resultados foram comparados pelo teste de Friedman com post hoc de Dunn. O nível de significância estabelecido foi de 5\%. Resultados: Não foram observadas diferenças estatisticamente significantes entre os valores medianos da FC de repouso (68bpm para 30\% e 60\%, 70bpm para $120 \% \mathrm{~s}$ ), FC pico $(107 \mathrm{bpm} \mathrm{para} 30 \%$, $103 \mathrm{em} 60 \%$ s e $100 \mathrm{bpm}$ em $120 \% \mathrm{~s})$ e $\Delta \mathrm{FC}\left(37 \mathrm{bpm}\right.$ para $30 \% \mathrm{~s}$, 35bpm em $60^{\circ} / \mathrm{s}$ e $27 \mathrm{bpm}$ para $\left.120^{\circ} \mathrm{s}\right)$, além do pico de torque $\left(209,5 \mathrm{~N} / \mathrm{m}\right.$ para $30^{\circ} /$ s, $217,2 \mathrm{~N} / \mathrm{m}$ em $60 \%$ e $210,6 \mathrm{~N} / \mathrm{m}$ para $120 \%$ s). A mediana dos valores de FC anteriores ao esforço foi semelhante aos de recuperação (67bpm). Conclusão: A magnitude de resposta da FC foi semelhante, durante atividade muscular excêntrica, indicando uma mesma sobrecarga cardíaca, independente da velocidade angular realizada.
\end{abstract}

Palavras-chave: freqüência cardíaca, exercício excêntrico, isocinético, ajustes cardiovasculares, extensores de joelho.

\section{ABSTRACT}

\section{Analysis of Heart Rate Response During Eccentric Isokinetic Exercise of Knee Extensor Muscle Group}

Objective: To investigate heart rate response magnitudes during eccentric isokinetic exercise of the knee extensor muscles, at different angular velocities. Method: Ten healthy and active young volunteers underwent maximum eccentric contraction of the extensor muscle group of the dominant knee. An isokinetic dynamometer was used at velocities of $30 \%$ s, $60 \%$ s and $120 \%$ s, randomly sequenced. The heart rate (HR) was obtained beat-by-beat, using a modified MC5 electrocardiogram lead, over 60s within the contraction, with five repetitions for each angular velocity, and over 120s after exercise effort. Mean HR for the 60s pre-exercise interval, peak HR attained at the end of exercise effort, HR variation $(\Delta \mathrm{HR})$ and peak torque were calculated. In addition, the pre-exercise mean HR over six minutes was compared with the recovery-period mean HR over six minutes. The results were compared using the Friedman and post hoc Dunn tests. The significance level was set at 5\%. Results: No statistically significant differences were observed among the median values of resting HR (68bpm for $30 \%$ and $60 \%$ s, $70 \mathrm{bpm}$ for $120^{\circ} / \mathrm{s}$ ), peak HR (107 bpm for 30\% $103 \mathrm{bpm}$ for $60 \%$ s and $100 \mathrm{bpm}$ for $120 \% \mathrm{~s}$ ), $\Delta \mathrm{HR}$ (37bpm for $30 \% \mathrm{~s}$, 35bpm for $60 \% \mathrm{~s}$ and $27 \mathrm{bpm}$ for $120 \%$ s) and peak torque $(209.5 \mathrm{~N} / \mathrm{m}$ for $30 \% \mathrm{~s}, 217.2 \mathrm{~N} / \mathrm{m}$ for $60 \%$ s and $210.6 \mathrm{~N} / \mathrm{m}$ for $120 \% \mathrm{~s})$. The median HR values before and after exercise effort were the same $(67 \mathrm{bpm})$. Conclusion: The HR response magnitudes during eccentric muscular activity were similar, which indicates that the cardiac overload was the same for all the angular velocities studied.

Key words: heart rate, eccentric exercise, isokinetic, cardiovascular response, knee extensor muscles. 


\section{INTRODUÇÃO}

A crescente utilização do dinamômetro isocinético na reabilitação ortopédica requer conhecimentos dos ajustes fisiológicos gerados por esse tipo de exercício ${ }^{1}$. Vários estudos tratam sobre os efeitos musculares do treinamento isocinético ${ }^{2,3,4}$, entretanto, poucas pesquisas se concentram na análise das respostas cardiovasculares geradas por esse tipo de exercício, principalmente durante a contração excêntrica ${ }^{5,6}$.

O exercício excêntrico tem sido empregado na prática clínica em fisioterapia para prevenção e reabilitação de danos musculoesqueléticos. Pelo fato de estar associado à maior capacidade de desenvolver força e ao menor consumo de oxigênio quando comparado com o exercício concêntrico, o treinamento excêntrico pode ser essencial para pacientes com limitada capacidade de exercício ${ }^{3}$.

Dessa forma, as respostas relacionadas ao aumento da demanda cardíaca durante o exercício excêntrico podem ser representadas pela análise da resposta da FC, uma vez que esta reflete indiretamente a quantidade de oxigênio transportado pelo sistema cardiovascular ${ }^{7}$.

O padrão e a magnitude de resposta da FC apresentam características típicas de acordo com o exercício executado. Estudos permitem concluir que o exercício concêntrico resulta em maior ativação cardiocirculatória que o excêntrico, apesar de produzir um nível de estiramento muscular e torque menores ${ }^{5}$.

Haennel et al. ${ }^{6}$ estudando as respostas cardiovasculares ao exercício isocinético concêntrico de flexão/extensão de joelho e de cotovelo, nas velocidades de $30^{\circ}, 90^{\circ}, 150^{\circ}$, observaram que o "stress" cardiovascular (freqüência cardíaca, pressão arterial média e resistência vascular periférica) associado a este tipo de exercício é independente da velocidade do movimento e proporcional à massa muscular ativa. Os autores referem ainda que a ação de grandes massas musculares ou a realização de exercícios em intensidades relativas mais altas promove significativo aumento da FC como resultado do maior grau de excitação de receptores musculares aferentes.

No entanto, poucos estudos se destinaram a pesquisar diferentes velocidades durante a contração excêntrica. Assim, o presente trabalho se justifica no sentido de trazer contribuições relativas às respostas da FC e observar a existência de sobrecarga cardíaca durante o exercício isocinético excêntrico de grupamento extensor do joelho, em diferentes velocidades angulares, o que permitirá orientações mais seguras quanto à realização deste tipo de exercício.

\section{MATERIAIS E MÉTODOS}

\section{Sujeitos}

Foram selecionados dez homens, com idade média de $22,3 \pm 2,4$ anos e valores médios de estatura, massa corporal e índice de massa corpórea de 180,5 $\pm 7 \mathrm{~cm}, 73,5 \pm 9 \mathrm{Kg}$ e $22,5 \pm 1,5 \mathrm{Kg} / \mathrm{m}^{2}$, respectivamente. Todos os voluntários eram saudáveis, não fumantes e com padrão ativo de vida, caracterizado por realização de atividade física aeróbia de natureza recreacional ou de lazer, com duração média de 3,75 horas semanais. Como critério de inclusão dos sujeitos no estudo, exigia-se não apresentarem alterações nos sistemas cardiovascular e musculoesquelético, avaliados por meio de anamnese, exames físico e postural, ECG convencional em repouso e durante teste ergométrico máximo. Além disso, os voluntários foram submetidos a exames clínicos e laboratoriais, como raio $\mathrm{X}$ de tórax, exame de urina, hemograma completo, colesterol (total e frações), triglicérides, ácido úrico, creatinina e uréia.

Os voluntários foram informados da natureza e propósito do estudo e assinaram um termo de consentimento para a realização dos testes. Este projeto foi aprovado pelo Comitê de Ética em Pesquisa da Universidade Federal de São Carlos $\left(n^{\circ} 045 / 03\right)$.

\section{Procedimento Experimental}

Os testes e procedimentos experimentais foram realizados no Ambulatório de Fisioterapia da Universidade Federal de São Carlos e no Laboratório de Fisioterapia Cardiovascular - NUPEF da UFSCar.

Os voluntários foram orientados para na véspera e no dia do teste não ingerirem bebidas alcoólicas e/ou estimulantes, não realizar atividade física extenuante, evitar refeição pesada até duas horas antes dos testes, dormir bem no dia anterior e trajar roupas leves.

O protocolo constou de um teste de exercício isocinético excêntrico máximo para grupo extensor do joelho dominante, no modo excêntrico-excêntrico, com cinco repetições em cada velocidade angular estudada, sendo $30 \%$ s, $60 \%$ s e $120 \%$ $\mathrm{s}$, ordenadas aleatoriamente por meio de sorteio. O joelho foi posicionado entre $45^{\circ}$ e $90^{\circ}$ de flexão, completando uma amplitude de movimento de $45^{\circ}$.

Precedendo a aplicação do protocolo, os voluntários foram submetidos a três séries de alongamento para os músculos isquiotibiais, quadríceps femoral e tríceps sural, e imediatamente após, pedalaram durante 5 minutos em uma bicicleta ergométrica estacionária, sem carga e mantendo uma velocidade de $25 \mathrm{Km} / \mathrm{h}^{8}$.

Os testes foram realizados utilizando-se um dinamômetro isocinético da marca BIODEX modelo System II, software v.4.5.

Para a realização do protocolo experimental, o sujeito foi posicionado no equipamento na posição sentada, com o quadril flexionado a $90^{\circ}$, alinhando o epicôndilo lateral do fêmur testado com o eixo de rotação do dinamômetro. O membro inferior testado ficou fixado no dispositivo do equipamento, 
mantido em dois centímetros acima do maléolo lateral do tornozelo, e estabilizado por uma correia de contenção. A coxa foi fixada no assento por uma correia passada sobre o terço distal da mesma. O tronco foi estabilizado por correias escapulares.

O voluntário permaneceu na postura sentada para que fosse realizada a tricotomia, limpeza e colocação de eletrodos de carbono ativado, auto-adesivos e descartáveis. Em seguida, FC era coletada batimento a batimento, a partir da derivação MC5 modificada (pólo negativo no manúbrio esternal, pólo positivo na região do $6^{\circ}$ espaço intercostal na linha hemiclavicular esquerda e o terra no $5^{\circ}$ espaço intercostal direito).

A FC foi captada por 6 minutos em repouso na posição sentada (condição controle). Durante os testes, por 60s em repouso pré-teste, durante o esforço e por 120s de recuperação, para cada velocidade estudada. Foi dado um intervalo entre cada teste de modo que a FC retornasse aos valores basais da condição controle. Após a realização de todos os testes, a FC foi captada no período de recuperação, por 6 minutos.

Para a captação e processamento dos sinais eletrocardiográficos foi utilizado um monitor cardíaco de 1 canal (TC -500, ECAFIX) acoplado a um conversor analógico digital Lab - PC + (National Instruments, Co), que constitui numa "interface” entre o monitor cardíaco e o microcomputador Pentium III. A partir da "interface”, o sinal analógico do ECG foi convertido em valores binários para o acesso no microcomputador, por meio de um programa de processamento dos sinais digitalizados ${ }^{9}$.

Durante todo o experimento foi solicitado aos voluntários que mantivessem respiração espontânea, que não realizassem contração de outros músculos que não os avaliados e que evitassem a realização conjunta de manobra de Valsalva, que foi controlada pela ausência de intensa bradicardia reflexa, observada por meio das respostas da FC na tela do computador, ao término de cada teste ${ }^{10}$.

Os testes foram realizados sempre no mesmo horário do dia, a partir das 18 horas. A temperatura ambiente foi mantida em 23,5 \pm 0,94 graus Celsius e a umidade relativa do ar foi de $62,7 \pm 2,58 \%$.

\section{Análise dos dados}

A partir dos dados da FC obtidos batimento a batimento, os valores numéricos considerados para cada um dos voluntários estudados foram:

- A média da FC durante os 6 minutos de repouso inicial, na posição sentada, pré-teste (condição controle);

- A média da FC durante os 6 minutos de recuperação, na posição sentada, pós-teste;

- A média da FC no período de 60s anteriores à realização do esforço;

- A FC pico durante o esforço;
- A variação da $\mathrm{FC}(\Delta \mathrm{FC})$, que corresponde à diferença entre FC pico e a média da FC dos 60s de repouso;

Foram coletados os valores de pico de torque $(\mathrm{N} / \mathrm{m})$, fornecido pelo laudo do equipamento, para cada velocidade, de todos os voluntários.

\section{Análise Estatística}

Os resultados foram apresentados graficamente em “Box-plot” contendo os valores da mediana, $1^{\circ}$ quartil (25\%), $3^{\circ}$ quartil (75\%), valores máximos e mínimos, “outliers” e extremos.

A análise dos dados não apresentou distribuição normal. Foi utilizado o teste estatístico não paramétrico de Friedman com post hoc de Dunn, uma vez que o nível de significância foi de $\alpha=5 \%$. Foi utilizado o aplicativo "Statistica for Windows, Release 5.1 Stat. Soft, Inc.2000-2001”.

\section{RESULTADOS}

Os valores medianos da média FC observados entre as condições: repouso 6 minutos pré-teste (controle), nos 60s pré-exercício nas velocidades de $30 \%$ s, $60 \%$ s, $120 \%$, e na condição de repouso 6 minutos após todos os testes (recuperação), foram respectivamente de 66 bpm, 68 bpm, 68 bpm, 70 bpm, 68 bpm. Não foram encontradas diferenças estatisticamente significantes entre os mesmos.

Este resultado indica que o intervalo entre cada teste foi suficiente para a FC retornar aos valores basais (condição controle), sendo assim não houve interferência da execução de um exercício em relação ao outro.

Os valores da FC pico durante o esforço, para cada velocidade angular executada, estão apresentados na Figura 1, onde se observa que não houve diferenças estatisticamente significantes entre estas (valores medianos em 30\% $=107$ bpm; em 60\% = 103 bpm; em 120\% = 100 bpm, p>0,05).

$\mathrm{Na}$ figura 1 também se encontram os valores das variações da FC durante o exercício nas três velocidades angulares realizadas; não houve diferenças estatisticamente significantes entre as velocidades (valores medianos em $30 \%$ $\mathrm{s}=37 \mathrm{bpm}$; em $60 \% \mathrm{~s}=36 \mathrm{bpm}$; em $120 \% \mathrm{~s}=28 \mathrm{bpm}$, $\mathrm{p}>0,05)$.

$\mathrm{Na}$ figura 2 observamos os valores de pico de torque $(\mathrm{N} / \mathrm{m})$ para cada velocidade; não houve diferença estatisticamente significante entre as velocidades (valores medianos de $30 \% \mathrm{~s}=209,5 \mathrm{~N} / \mathrm{m} ; 60 \% \mathrm{~s}=217,2 \mathrm{~N} / \mathrm{m} ; 120 \%$ $\mathrm{s}=210,6 \mathrm{~N} / \mathrm{m})$.

Considerando-se que no experimento foi utilizado o mesmo número de repetições, observamos que na velocidade angular mais baixa $(30 \%)$, os voluntários realizaram o exercício proposto em maior tempo (média de 23,5s), na velocidade de $60 \%$ s o tempo médio de execução foi de 15 
segundos, e na velocidade mais alta (120\%) o tempo gasto para a realização do exercício foi menor (média de 10s).

O padrão de resposta da FC nas três velocidades angulares executadas por um dos voluntários está ilustrado na Figura 3. Ao início da contração, observamos um súbito aumento da FC, que continuou aumentando durante o período de realização do exercício. Esta declinou progressivamente após a interrupção do mesmo, retornando aos valores basais. Este padrão de resposta foi observado em todos os voluntários, nas três velocidades angulares estudadas.
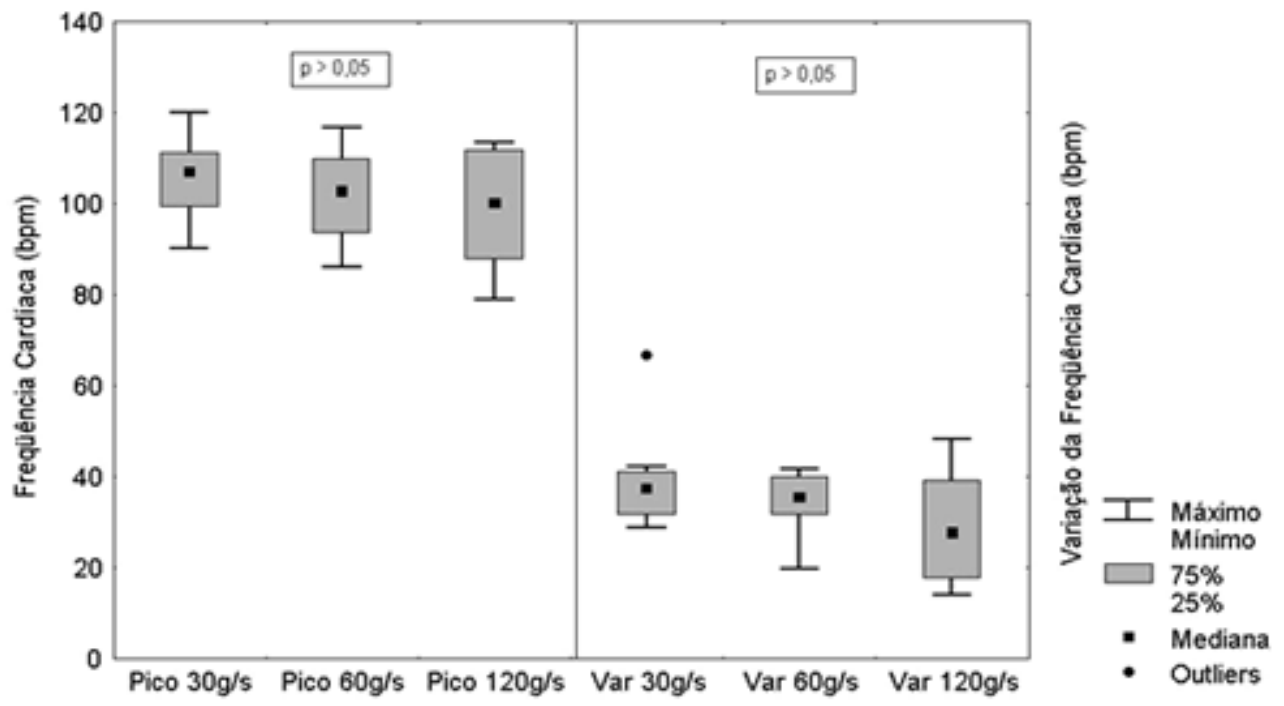

Figura 1. Valores da freqüência cardíaca (FC) pico e da variação da FC, em batimentos por minuto (bpm), durante o exercício isocinético excêntrico de grupamento extensor de joelho, nas velocidades angulares de 30, 60 e 120 graus por segundo, dos voluntários estudados. Nível de significância de $\alpha=5 \%$.

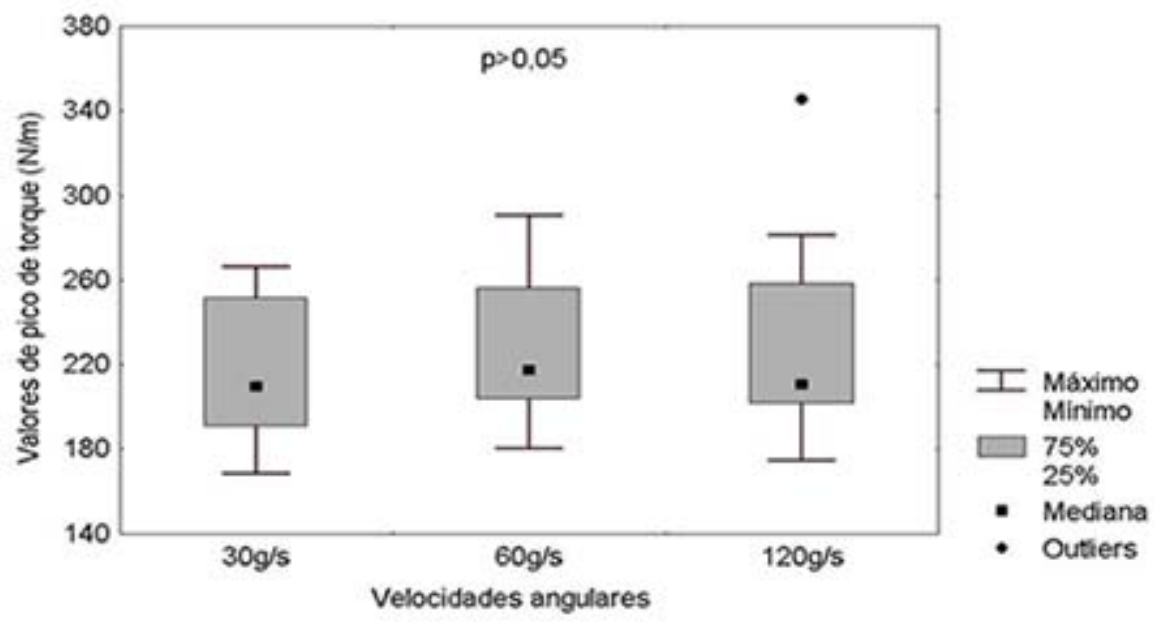

Figura 2. Valores de pico de torque (N/m) nas velocidades angulares de 30, 60, 120 graus por segundo. Nível de significância de $\alpha=5 \%$.

\section{DISCUSSÃO}

Com intuito de minimizar possíveis influências na resposta da FC, a amostra foi padronizada com relação às características antropométricas, idade, sexo, estilo de vida, bem como foram controladas as condições ambientais (temperatura, umidade relativa do ar), e o período do dia em que foram realizados os experimentos ${ }^{11}$.

Existem vários estudos ${ }^{12,13,14}$ relacionando as respostas de diferentes variáveis cardiovasculares, como pressão arterial (PA), freqüência cardíaca (FC) e resistência vascular periférica (RVP) entre o exercício isocinético concêntrico e excêntrico realizados na mesma velocidade angular. Entretanto, pouca 


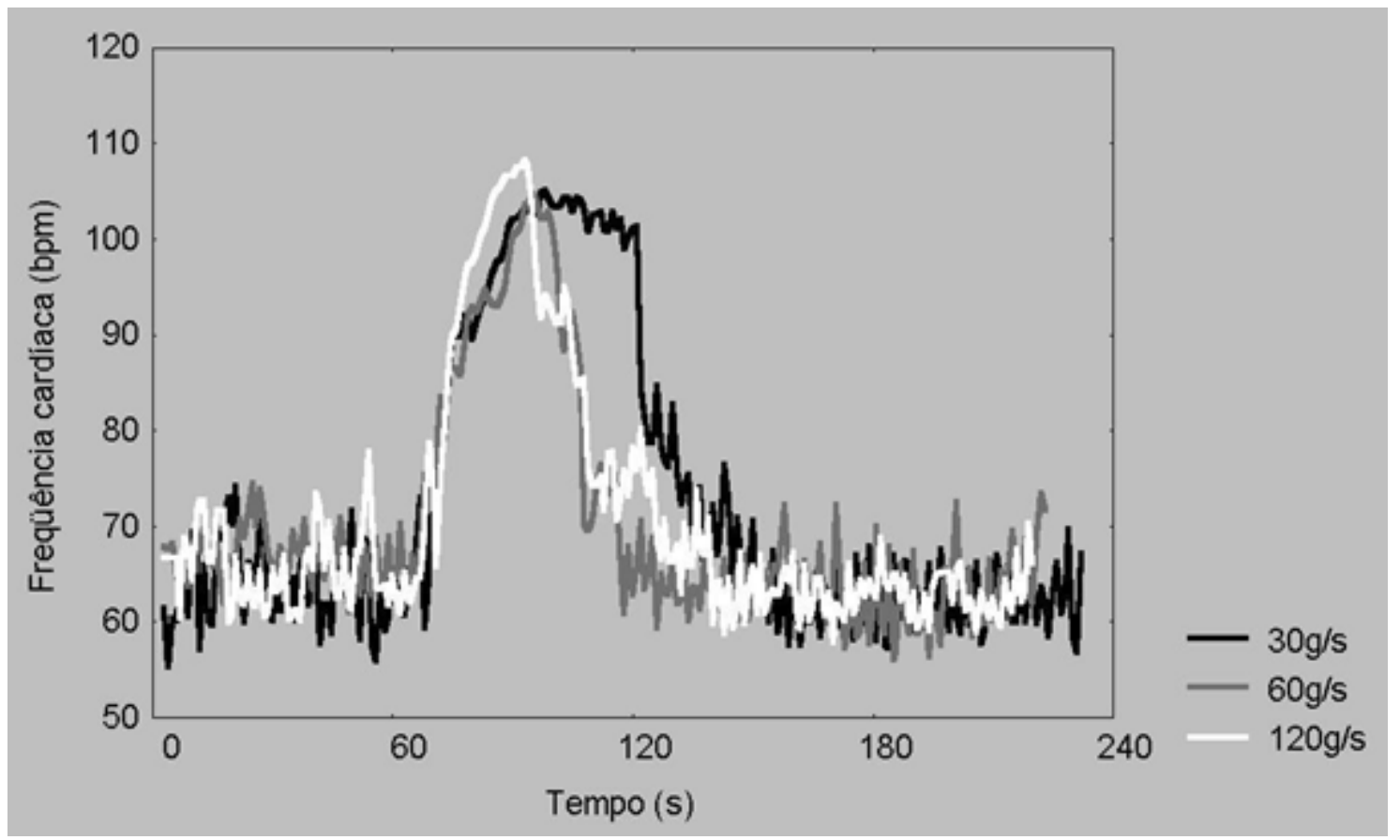

Figura 3. Comportamento da freqüência cardíaca (FC) durante o exercício isocinético excêntrico do grupamento extensor do joelho nas velocidades angulares de 30, 60 e 120 graus por segundo, durante os 60 segundos de repouso, durante o exercício e nos 120 segundos de recuperação, para um dos voluntários estudados.

atenção é direcionada para os efeitos da contração isocinética excêntrica isoladamente, em diferentes velocidades, sendo este, portanto o objeto de estudo da presente investigação.

Durante o exercício isocinético excêntrico de joelho realizado em diferentes velocidades angulares, os valores de FC de pico observados foram semelhantes. Da mesma forma, ao analisarmos a variação da FC durante este tipo de exercício, observamos que a magnitude da resposta da FC não diferiu nas diferentes velocidades angulares realizadas.

Haennel et al. ${ }^{6}$ sugerem que a velocidade do movimento não exerce influência sobre os ajustes cardiovasculares durante a realização de exercício isocinético concêntrico realizado na intensidade máxima. Segundo os autores, as respostas cardiovasculares são proporcionais à massa muscular em atividade, e não são influenciadas pelas diferentes velocidades angulares.

Nossos resultados são concordantes com os de Haennel et al. ${ }^{6}$ em relação à independência da velocidade sobre a resposta da FC, embora estes autores tenham estudado o exercício isocinético concêntrico em diferentes velocidades angulares. Cabe ressaltar que, neste trabalho a massa muscular ativa foi a mesma para todas as velocidades angulares realizadas, e assim não exerceu influência nas respostas obtidas.

No presente estudo, considerando-se que o número de repetições foi mantido para as 3 velocidades, encontramos um tempo maior de exercício na velocidade de $30 \%$ s, apesar dos voluntários apresentarem valores de FC semelhantes para as três velocidades angulares estudadas. Dessa forma, observamos semelhante sobrecarga cardíaca independente do tempo gasto para a realização do exercício e da velocidade angular executada.

No exercício isocinético concêntrico, quanto menor a velocidade angular, maior é a sobrecarga muscular, ou seja, mais torque é gerado, podendo influenciar mais na sobrecarga cardíaca ${ }^{5}$. No exercício isocinético excêntrico por sua vez, ainda não está muito clara a relação entre velocidade angular e torque ${ }^{15}$. Neste trabalho, não houve diferenças nos valores de torque entre as velocidades estudadas, sendo assim, isto pode ter contribuído para a ausência de diferenças na resposta da FC em diferentes velocidades.

\section{Padrão de resposta da FC ao exercício isocinético excêntrico}

Têm sido descrito na literatura que durante o exercício físico dinâmico (EFD) realizado em protocolos descontínuos, a resposta da FC apresenta característica típica ${ }^{16,17}$. Ocorre um aumento súbito nos 10-20 segundos iniciais do exercício, em decorrência da retirada da atividade vagal no nodo sinoatrial do coração, denominado componente rápido de elevação da FC. Em níveis submáximos de EFD, logo após a fase de incremento rápido da $\mathrm{FC}$ o nervo vago retoma sua atividade no coração, resultando numa diminuição da FC ou uma 
tendência ao steady state nos valores da mesma. Em cargas elevadas e acima de um minuto de realização do EFD pode ocorrer um aumento mais lento da FC que será dependente da predominância da estimulação simpática sobre o nodo sinoatrial, caracterizando o incremento lento da $\mathrm{FC}^{17,18,19}$.

Durante o exercício isométrico, está bem esclarecido que ocorre elevação na FC, caracterizada por uma reposta inicial rápida, atribuída a inibição da atuação vagal sobre o nodo sino-atrial, que ocorre até os 10s iniciais do exercício. Dependendo do percentual de contração voluntária máxima realizada e do tempo de duração da mesma, a FC mantémse elevando gradualmente, sendo que esta elevação lenta é predominante devido à estimulação simpática ${ }^{20}$.

No presente trabalho, ao estudarmos a resposta da FC ao exercício isocinético excêntrico que é considerado um tipo de exercício dinâmico, observamos resultados similares aos apresentados na literatura ${ }^{16,20}$. Assim, ao início do exercício, observamos um súbito aumento da FC devido à retirada do tônus vagal atuante sobre o nodo sino-atrial, embora a dinâmica de liberação vagal pareça ser mais lenta. Durante os testes em que o tempo despendido foi maior (25s) a FC continuou aumentando, caracterizando ainda a participação da retirada vagal. Isto se baseia em estudo realizado com bloqueio farmacológico que infere que a inibição vagodependente pode ocorrer até 30 s de exercício ${ }^{17}$. Ao término do esforço, a FC declinou progressivamente até retornar aos valores basais. Esse comportamento da FC foi semelhante em todas as velocidades angulares executadas.

A literatura refere que há uma redução na atividade muscular voluntária em humanos durante a atividade excêntrica máxima ${ }^{21}$. Paddon-Jones et al. ${ }^{22}$ relatam que essa redução é predominante durante contrações excêntricas rápidas. Para Seger e Thorstensson ${ }^{23}$, esse mecanismo compreende os componentes neurais centrais e periféricos, que são ativados quando grandes forças musculares são produzidas, com o objetivo de prevenir danos nas estruturas articulares. O feedback gerado neste processo por órgãos periféricos, como fusos musculares, receptores articulares e órgão tendinoso de Golgi e nocioceptores, pode exercer importante papel na limitação do desenvolvimento de força muscular.

Poderíamos sugerir a influência deste mecanismo na ativação muscular e também na resposta cardíaca gerada em nossos testes executados na velocidade mais alta $(120 \%$ s), se fosse comprovado que todos os voluntários executaram atividade máxima, pois não encontramos diferenças entre os valores de pico de torque nas diferentes velocidades. Desse modo, o fato de termos observado semelhante sobrecarga cardíaca em todas as velocidades estudadas não nos permite explicar nossas respostas com base neste mecanismo, destacando a necessidade de mais estudos nesta área.

Podemos concluir que a magnitude de resposta da FC foi semelhante durante o exercício excêntrico do grupamento extensor de joelho, nas velocidades de $30 \%$ s, $60 \%$ s e $120 \%$ s, com o mesmo número de repetições, indicando uma mesma sobrecarga cardíaca independente da velocidade angular realizada.

Assim, o presente trabalho contribui para a prática clínica no sentido de permitir orientações mais seguras quanto à realização deste tipo de exercício, considerando-se as condições experimentais estudadas.

\section{REFERÊNCIAS BIBLIOGRÁFICAS}

1. Sherman W, Pearson D, Phylery M, Costill D, Habansky A, Vogelsang D. Isokinetic rehabilitation after surgery. Am J Sports Med 1982; 10: 155-61.

2. Lesmes GR, Costill DL, Coyle EF, Fink WJ. Muscle strength and power changes during maximal isokinetic training. Med Sci Sports Exerc 1978; 10: 266-69.

3. Kellis E, Baltzopoulos V. Isokinetic eccentric exercise. Sports Med 1995; 19(3): 202- 22.

4. Proske U, Morgan Dl. Muscle damage from eccentric exercise: mechanism, mechanical signs, adaptation and clinical applications. J Physiol 2001; 573(2): 333-45.

5. Horstmann T, Mayer F, Fisher J, Maschmann J, Rocker K, Dickhuth Hh., The cardiocirculatory reaction to isokinetic exercises in dependence on the form of exercise and age. Int $\mathrm{J}$ Sports Med 1994; 15: S50-S55.

6. Haennel RG, Snydmiller GD, Teo KK, Greenwood PV, Quinney HÁ, Kappagoda Ct. Changes in blood pressure and cardiac output during maximal isokinetic exercise. Arch Phys Med Rehabil 1992; 73: 150-55.

7. Gallo Jr L, Maciel BC, Marin-Neto JA, Martins LEB., Lima-Filho EC, Golfetti R, et al. Control of heart rate during exercise in health and disease. Braz J Med Biol Res 1995; 28: 1179-84.

8. Guaratini MI. Confiabilidade e precisão de medida para testereteste do dinamômetro isocinético Biodex [dissertação]. São Carlos (SP): UFSCar; 1999.

9. Silva E, Catai AM, Trevelin LC, Guimarães JO, Silva JR. LP, Silva LMP, et al .Design of computerized system to evaluate the cardiac function during dynamic exercise. Phys Med Biol 1994; 33: 409 .

10. Marães VRFS, Santos MDB, Catai AM, Moraes FR, Oliveira L, Gallo Jr L, Silva E. Modulação do sistema nervoso autônomo na resposta da freqüência cardíaca em repouso e à manobra de Valsalva com o incremento da idade. Rev Bras Fisioterapia 2004; 8(2): 97-103.

11. Catai AM, Chacon-Mikahil MPT, Martinelli FS, Forti VAM, Silva E, Golfetti R, et al. Effects of aerobic exercise training on heart rate variability during wakefulness and sleep and cardiorespiratory responses of young and middle-aged healthy men, Braz J Med Biol Res. 2002; 35(6): 741-752.

12. Garcia APU. Variabilidade da freqüência cardíaca e sinal eletromiográfico do vasto lateral de ciclistas durante exercício físico dinâmico em 60 e 80 rotações por minuto [dissertação]. São Carlos (SP): UFSCar; 2003. 
13. Kellis E, Baltzopoulos V. Muscle ativation differences between eccentric and concentric isokinetic exercise. Med Sci Sports Exerc 1997; 30 (11): 1616-23.

14. Mayer F, Axmann D, Horstmann T, Niess A, Striegel H, Ruf J, Dickhuth HH. Metabolic and cardiocirculatory reactions after concentric and eccentric exercise of the shouder. Int J Sports Med 1999; 20: 527-31.

15. Overend TJ, Versteegh TH, Thompson e, Birmingham TB, Vandervoort AA. Cardiovascular stress associated with concentric and eccentric isokinetic exercise in young and older adults. J Gerontol 2000; 55A(4): B177-82.

16. Kellis E, Baltzopuolos V. Isokinetic eccentric exercise. Sports Med 1995; 19(3): 202-22.

17. Gallo Jr L, Maciel BC, Marin Neto JA, Martins LEB. Ajustes cardiovasculares ao exercício físico: efeitos do treinamento aeróbio. Med 1999; 23(2): 101-06.

18. Catai AM. Adaptações cardiorrespiratórias em atletas: estudo em diferentes fases do treinamento físico [dissertação]. Campinas (SP): UNICAMP; 1992.

19. Maciel BC, Gallo Jr L, Marin Neto JA, Lima Filho EC, Terra Filho J, Manço JC. Parassympathetic contribuition to bradicardia induced by endurance training in man. Cardiovasc Res 1985; 19: 642-48.

20. Menegon FA. Variações e relações entre freqüência cardíaca, eletromiografia e geração de torque desenvolvidos durante contração voluntária máxima de extensão e flexão do cotovelo e do joelho [dissertação]. São Carlos (SP): UFSCar; 2003.

21. Rutherford Om, Purcell C, Newham Dj. The human force: velocity relationship activity in the knee flexor and extensor muscles before and after eccentric practice. Eur J Appl Physiol 2001; 84: 133-40.

22. Paddon-Jones D, Levenitt M, Lonergan A, Abernethy P., Adaptation to chronic eccentric exercise in humans: the influence of contraction velocity. Eur J Appl Physiol 2001; 85: 466-71.

23. Seger Jy, Thorstensson A. Electrically evoked eccentric and concentric torque-velocity relantionships in human knee extensor muscles. Acta Physiol Scand 2000; 169: 63-69. 